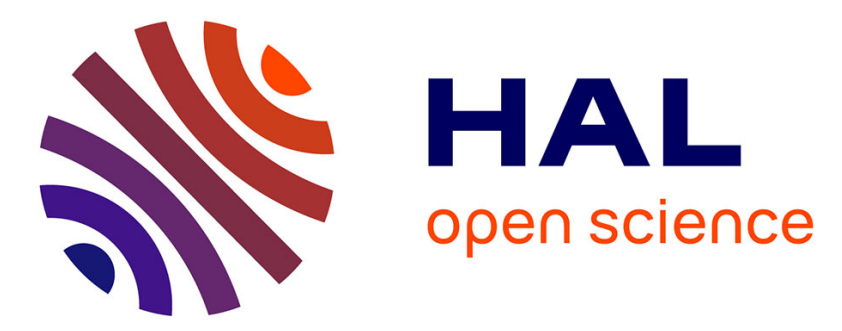

\title{
Arbitrage with Fixed Costs and Interest Rate Models
}

Elyès Jouini, Clotilde Napp

\section{To cite this version:}

Elyès Jouini, Clotilde Napp. Arbitrage with Fixed Costs and Interest Rate Models. Journal of Financial and Quantitative Analysis, 2006, pp.889-913. halshs-00176496

\section{HAL Id: halshs-00176496 \\ https://shs.hal.science/halshs-00176496}

Submitted on 3 Oct 2007

HAL is a multi-disciplinary open access archive for the deposit and dissemination of scientific research documents, whether they are published or not. The documents may come from teaching and research institutions in France or abroad, or from public or private research centers.
L'archive ouverte pluridisciplinaire HAL, est destinée au dépôt et à la diffusion de documents scientifiques de niveau recherche, publiés ou non, émanant des établissements d'enseignement et de recherche français ou étrangers, des laboratoires publics ou privés. 


\title{
Arbitrage With Fixed Costs" and Interest Rate Models
}

\author{
Elyès JOUINI* $\quad$ Clotilde NAPP ${ }^{\dagger}$
}

June 26, 2007

${ }^{*}$ Corresponding author. CEREMADE, Université de Paris IX Dauphine, Place du Maréchal de Lattre de Tassigny, 75116 Paris. e-mail : jouini@ceremade.dauphine.fr

${ }^{\dagger}$ CEREMADE, Université de Paris IX Dauphine, Place du Maréchal de Lattre de Tassigny, 75116 Paris and CREST. e-mail : napp@ceremade.dauphine.fr. We thank Hedi Kallal for helpful discussions and comments as well as for careful reading. We also thank NFA 2002 Congress participants and in particular D. Feldman. 


\title{
Arbitrage With "Fixed Costs" and Interest Rate Models
}

\begin{abstract}
In this paper, we study securities market models with "fixed costs." We characterize the absence of arbitrage opportunities and we provide fair pricing rules. We then apply these results to extend some popular interest rate and option pricing models, which present arbitrage opportunities in the absence of "fixed costs."

In particular, we prove that the quite striking result obtained by Dybvig, Ingersoll and Ross (1996), which asserts that, under the assumption of absence of arbitrage, long zero-coupon rates can never fall, is no longer true in models with "fixed costs," even arbitrarily small ones. For instance, models where the long-term rate follows a diffusion process are arbitrage-free in the presence of "fixed costs" (including arbitrarily small ones). We also rationalize models with partially absorbing or reflecting barriers on the price processes. In particular, we propose a version of the Cox, Ingersoll, and Ross (1985) model which, as in Longstaff (1992), produces yield curves with realistic humps but does not assume an absorbing barrier for the short-term rate. This is made possible by the presence of (even arbitrarily small) "fixed costs."
\end{abstract}

Keywords arbitrage - fixed costs - contingent claims pricing - interest rate models - long zerocoupon rates - Dybvig, Ingersoll and Ross - Brennan and Schwartz - barrier models. 


\section{Introduction}

In this paper, we study securities market models with "fixed costs." We characterize the absence of arbitrage opportunities and we provide fair pricing rules. We then apply these results to extend some classical interest rate and option pricing models which present arbitrage opportunities in the absence of "fixed costs."

Transaction costs are usually said to be fixed if they are constant regardless of the size of the transaction. In this paper, we study more general "fixed costs" structures where the cost of transacting has a fixed component, i.e., is bounded away from zero independently of the quantity traded. Some results will more specifically focus on "bounded fixed costs" structures, for which transaction costs are bounded (below and above) regardless of the size of the transaction or on "vanishing fixed costs" structures, i.e., transaction costs structures such that the marginal cost tends to zero when the transaction size increases. Note that "bounded fixed costs" structures are a specific case of "vanishing fixed costs" structures.

As underlined by Brennan (1975), such "fixed costs" structures capture reasonably well the basic features of the commission structure of many stock exchanges which involve a stated amount plus a declining percentage of the value of the transaction. In fact, investors incur many different fees and charges that depend, among other things, on the traded volume, traded amount, traded asset, and the total fee is in general bounded below by a given amount that corresponds to the ticket charges that have to be paid for each transaction regardless to the size of that transaction and bounded above. For instance, on the GLOBEX clearing system where fees are capped (for trades executed for members trading within their division for their own account) at $\$ 50$ per day per product, per operator. ${ }^{1}$ We refer to Jones (2002) for a general

\footnotetext{
${ }^{1}$ More details on the clearing and execution fees are provided on the Chicago Mercantile Exchange website
} 
description of the trading costs structure on the NYSE accross the 20th century.

Note that such a "fixed costs" structure is not limited to the modelization of clearing or trading fees imposed by stock exchanges. Indeed, as underlined by Leape (1987),

"acquiring assets virtually always incurs financial charges such as brokerage fees, which in general have a fixed costs component (...). Even in the cases in which such costs appear proportional to the amount invested, there are typically minimum investment requirements which are equivalent to fixed costs of trading. The costs of acquiring assets also include the opportunity costs and information costs associated with analyzing new assets. Such costs are truly costs of participating in particular markets and are independent of the amount invested."

In fact, our "fixed costs" structure can model brokerage fees, holding costs, fixed investment taxes to gain access to a market (such as a foreign market), operational and processing costs that typically exhibit strong economies of scale (e.g. through automation), "fixed costs" involved in setting up an office and obtaining access to information, the opportunity cost of looking at a market or of doing a specific trade and brokerage arrangements where marginal fees go to zero beyond a given volume that is reset periodically (a common practice in the industry).

Other frictions studied in the literature include transaction costs that are proportional to the quantity of asset traded. Without minimizing the relevance of this type of frictions, the "fixed costs" specification analyzed in this paper is of importance for three major reasons developed by Viard (1995) :

"First as Leape (1987) argued, a number of costs associated with purchasing and

(http://www.cme.com/clr/clring/fees/equtyclrng1867.html) 
holding assets approximately satisfy the fixed-costs specification (...)

Second, such costs are more analytically challenging than proportional costs. The latter can be easily accomodated in the standard CAPM framework with little additional analysis, because such costs simply reduce the net rate of return. ${ }^{2}$

Third, fixed costs offer a simple and plausible explanation for the observation that many investors hold exactly zero of most available assets; although proportional costs would alter an investor's holding of any particular asset, they generally would not drive such holdings to zero."

We find that the absence of arbitrage opportunities in models with "bounded fixed costs" (or more generally with "vanishing fixed costs") is equivalent to the existence of a family of nonnegative state-price densities compatible with the asset price processes. The only difference with the frictionless setting lies in the fact that these state-price densities may be nonnegative instead of positive. When the fixed costs are neither bounded nor vanishing, we still have that the existence of nonnegative state price densities implies the absence of arbitrage opportunity, but the converse implication does not necessarily hold. Remark that a zero state price density for some states of the world does not mean that investors might have consumption for free in those states since they always incur a positive fixed cost. Furthermore, we define fair pricing rules as those that are arbitrage-free and lie below the superreplication cost. Indeed, no rational agent would pay more than its superreplication cost for a contingent claim since there is a cheaper way to achieve at least the same payoff using a trading strategy. We then show that the only fair pricing rules are those that are equal to the sum of a "fixed cost" function and

\footnotetext{
${ }^{2}$ Although this is challenging in a dynamic setting (see Jouini and Kallal (1995) for a characterization of the no-arbitrage in a proportional transaction costs framework).
} 
of a linear pricing rule associated to one of the nonnegative state-price densities given by the absence of arbitrage. While we focus on discrete time models, the characterization of the absence of arbitrage opportunity remains essentially the same in a continuous time setting. However, as usual in continuous time and for technical reasons, the concept of no arbitrage is replaced by the one of no free-lunch, and the boundedness condition on the fixed costs structure has to be modified accordingly (see Jouini, Kallal and Napp, 2001).

The main impact of the introduction of "fixed costs," in discrete time as well as in continuous time, is the enlargement of the set of possible state-price densities from the positive ones to the nonnegative ones. The absence of arbitrage opportunities in a "fixed costs" framework is therefore weaker than in a frictionless model and some models that contain arbitrage opportunities in the absence of frictions might become arbitrage-free with the introduction of "fixed costs" including arbitrarily small ones. This enables us in Section 3, in a discrete time setting, to rationalize some model specifications that are usually rejected for arbitrage considerations, but have other desirable properties. The discrete time framework permits to provide intuitions about how things work and to see exactly how the fixed costs and the 0-weights in the state price densities rationalize the models under consideration.

In particular, we prove that the quite striking result obtained by Dybvig, Ingersoll and Ross (1996), which asserts that, under the assumption of absence of arbitrage, long zero-coupon rates can never fall, is no longer true in models with "fixed costs," even arbitrarily small. In particular, we consider discrete time versions of the classical Brennan and Schwartz (1979) model. Based on the pure expectation theory, this model assumes a diffusion behavior for long-term rates, ${ }^{3}$ and is

\footnotetext{
${ }^{3}$ Long rates are defined as the limit zero-coupon rate when maturity goes to infinity. It does not correspond to what is usually called long rate on the markets, namely the rate associated to the longest marketed maturities (e.g. 30 years).
} 
therefore incompatible with the absence of arbitrage result given by Dybvig, Ingersoll and Ross (1996). We show that such a model is now compatible with the absence of arbitrage (with "fixed costs," even arbitrarily small). This enables to reconcile arbitrage conditions and the empirical observations that do not seem to reject Brennan and Schwartz (1979) model (see Chan et al., 1992). In the same spirit, we also provide examples of term structure models adapted from Ho and Lee (1986) where the long-term zero-coupon rates can both increase and decrease.

"Fixed costs" also permit us to rationalize models with partially absorbing or reflecting barriers on the asset price process. In a frictionless framework, such models obviously admit arbitrage opportunities but they are arbitrage free in the presence of "fixed costs," including arbitrarily small ones. In addition, we show that in such models the price of a call option is equal to the price of a barrier option in a model without any barrier. We also propose a discrete-time version of a Cox, Ingersoll and Ross (1985) model which, as in Longstaff (1992), produces yield curves with realistic humps. However, our model does not assume an absorbing barrier for the short rate, but instead assumes "fixed costs."

There is an existing body of literature that studies fixed transaction costs: Duffie and Sun (1990), Grossman and Laroque (1990) and Morton and Pliska (1995), among others, study the optimal portfolio problem with transaction fees that are proportional to the size of the overall portfolio (as opposed to the size of the specific transaction). In the same spirit, Luttmer (1999) adresses the empirical problem of the required level of transaction costs for observations on consumption choices to be consistent with data on asset returns. Brennan (1975), Goldsmith (1976), Levy (1978), Mayshar $(1979,1981)$, Leape (1987) and Viard (1995) consider a CAPM model with "fixed costs" in order to propose an explanation to the empirical evidence of limited diversification: households hold a very limited set of assets. Jouini, Kallal and Napp (2001) study 
the characterization of the assumption of no-free-lunch as well as viability issues in securities market models with "fixed costs" in a continuous time framework.

The paper is organized as follows. Section 2 presents the absence of arbitrage characterization in models with "fixed costs," and provides a general description of fair pricing rules. Section 3 provides applications of these results to interest rate models. All proofs are in the Appendix.

\section{Models with "Fixed Costs"}

\section{A. Arbitrage in Models with "Fixed Costs"}

\section{Formulation of the Market Model}

The model to be studied here is the so-called event-tree model and the reader, familiar with such a framework, may skip the first paragraph.

A finite set $\Omega$ of states of the world is specified and fixed. Also specified is a time horizon $T$ which is the terminal date for all economic activity under consideration. The information arrival is given by a family of increasingly finer partitions $\left\{F_{0}, \ldots, F_{T}\right\}$ of $\Omega$ such that $F_{0}=\{\Omega\}$ and $F_{T}=\{\{\omega\}: \omega \in \Omega\}$. A date $t$-node is an element of $F_{t}$. We denote by $\Sigma_{t}$ the set of all date $t$-nodes and for $\sigma_{t} \in \Sigma_{t}, f\left(\sigma_{t}\right)=\left\{\sigma_{t+1} \in \Sigma_{t+1}: \sigma_{t+1} \subset \sigma_{t}\right\}$ can be interpreted as the set of the immediate successors of the date $t$-node $\sigma_{t}$.

There are $n+1$ assets and for a date $t$-node $\sigma_{t}$, we denote by $Z^{k}\left(\sigma_{t}\right)$ the price at time $t$ and in the node $\sigma_{t}$ of asset $k$. We assume that $Z^{0} \equiv 1$ : this amounts to assuming the existence of an asset called a numéraire, i.e. with positive prices at every time, and considering discounted price processes.

In this framework, a portfolio is described by a vector $\theta \in \mathbb{R}^{n+1}$ where $\theta_{k}$ is the quantity of asset $k$ held by the investor. A portfolio strategy is then an adapted process $\left(\theta_{t}\right)_{t=0, \cdots, T}$ where $\theta\left(\sigma_{t}\right)$ is the portfolio held at date $t$ in the event $\sigma_{t}$. 
Each time investors revise their portfolio of risky assets, they pay a given transaction cost. The amount paid might depend on the traded assets, the quantity of assets that are traded, the traded amount as well as on the portfolio composition and on previous trades. For a given portfolio strategy $\left(\theta_{t}\right)_{t=0, \cdots, T}$, the transaction cost paid by the investors at date $t$ and in the node $\sigma_{t}$ is given by $c^{\theta}\left(\sigma_{t}\right)$. We also denote by $C_{t}^{\theta}\left(\sigma_{t}\right)$, the total transaction costs paid by the investor up to time $t$. We shall impose the following conditions :

- For a given strategy $\theta$, the process $c^{\theta}$ is nonnegative and adapted to the information structure $\left\{F_{0}, \ldots, F_{T}\right\}$,

- if there is a trade at date $t$ and in the state of the world $\sigma_{t}$ (i.e. $\theta_{t}\left(\sigma_{t}\right) \neq \theta_{t-1}\left(\sigma_{t-1}\right)$ for $\sigma_{t-1} \in \Sigma_{t-1}$ such that $\left.\sigma_{t} \in f\left(\sigma_{t-1}\right)\right)$ then $C_{t}^{\theta}\left(\sigma_{t}\right)>\mathbf{c}$ where $\mathbf{c}$ is a given positive minimum transaction cost.

The first condition means that the "fixed costs" are nonnegative and depend only on present and past information and the second condition means that investors have to pay a given minimum amount at least once, in order to trade.

We shall say that the "fixed costs" are vanishing if

- for any strategy $\theta$, any date $t$ and any date- $t$ node $\sigma_{t}$, we have $\lim _{\lambda \rightarrow \infty} \frac{c^{\lambda \theta}\left(\sigma_{t}\right)}{\lambda} \rightarrow 0$.

This condition reflects the declining marginal rate of the transaction costs structure. It is in particular satisfied when the transaction costs are bounded.

Many transaction costs structures are taken into account with this modelization

- clearing fees and brokerage arrangements where the amount to be paid is a function of the traded amount and where marginal fees go to zero beyond a given amount. We have then 
$c_{t}^{\theta}=\sum_{k=1}^{n} f^{k}\left(\left(\theta_{t}^{k}-\theta_{t-1}^{k}\right) Z_{t}^{k}\right)$ where $f^{k}$ is the fees structure associated with asset $k$ and satisfies $f^{k}(x)>\mathbf{c}$ for $x \neq 0$ and $\lim _{\lambda \rightarrow \infty} \frac{f^{k}(\lambda x)}{\lambda} \rightarrow 0$,

- trading fees and brokerage arrangements where the amount to be paid is a function of the quantity of assets that are traded and where marginal fees go to zero beyond a given volume. We have then $c_{t}^{\theta}=\sum_{k=1}^{n} f^{k}\left(\theta_{t}^{k}-\theta_{t-1}^{k}\right)$ where $f^{k}$ is the fees structure associated with asset $k$ and satisfies the same conditions as above,

- holding costs where the amount to be paid is a function of the quantity of assets held in the portfolio. We have then $c_{t}^{\theta}=\sum_{k=1}^{n} f^{k}\left(\theta_{t}^{k}\right)$ where $f^{k}$ still satisfies the same conditions as above,

- fixed investment taxes to gain access to a market or "fixed costs" involved in setting up an office and obtaining access to information where the amount is paid once before the first access to that market. Let us denote by $C^{k}$ the amount to be paid in order to have a later access to the $k$-th market. We have then $C^{\theta}\left(\sigma_{t}\right) \geq C^{k}$ at any date where $\theta_{t}^{k}\left(\sigma_{t}\right) \neq \theta_{t-1}^{k}\left(\sigma_{t-1}\right)$ for $\sigma_{t-1} \in \Sigma_{t-1}$ such that $\sigma_{t} \in f\left(\sigma_{t-1}\right)$

- operational and processing costs that exhibit strong economies to scale where the paid amount might be a function of the traded amount or the traded quantity with a possible fixed initial amount. The costs structure is then a combination of the structures presented above.

In all the examples above we have assumed that there is no cost associated to transactions on the riskless asset denoted by 0 . In the case of transaction costs, this assumption is made without any loss of generality since any trade on the riskless asset implies a trade on at least 
one other asset. In the case of holding costs, this assumption means that there is no holding fee on the riskless asset. Such an assumption is made in order to make the arguments clearer and can be relaxed without difficulties.

\section{Arbitrage Opportunities}

We can now introduce the notion of arbitrage. As usual, an arbitrage opportunity is a plan that yields through some combination of buying and selling securities, a positive gain in some circumstances without a countervailing threat of loss in other circumstances. More precisely,

Definition 1. A two-period arbitrage opportunity with "fixed costs" consists of a portfolio ${ }^{4} \theta$ such that

$$
\begin{gathered}
\sum_{k=0}^{n} \theta_{k} Z^{k}\left(\sigma_{t}\right)+c^{\theta}\left(\sigma_{t}\right) \leq 0 \\
\text { and } \sum_{k=0}^{n} \theta_{k} Z^{k}\left(\sigma_{t+1}\right)-c^{\theta}\left(\sigma_{t+1}\right) \geq 0 \text { for all } \sigma_{t+1} \in f\left(\sigma_{t}\right)
\end{gathered}
$$

with at least one strict inequality, for some $t$ and some $\sigma_{t}$ in $\Sigma_{t}$.

Before going any further, we provide some intuition of how things work and illustrate our results. A strategy that leads, exclusive of transaction costs, to a nonnegative and nonzero net gain which is zero in some states, would be an arbitrage opportunity in the frictionless framework, but is not an arbitrage in the "fixed costs" setting. Indeed, since positive transaction costs have to be paid, the net gain (inclusive of transaction costs) becomes negative in some states. For instance, a corporate bond and a government bond with the same characteristics can have the same price in a model with "fixed costs" even if the corporate bond has a positive probability of

\footnotetext{
${ }^{4}$ Strictly speaking, the transaction costs process $c^{\theta}$ is only defined for strategies $\theta$ and not for portfolios. However, for a give date-t node $\sigma_{t}$ a portfolio $\theta$ can obviously be identified with the strategy that consists in buying that portfolio at date $t$ in the event $\sigma_{t}$ and selling it at date $t+1$.
} 
default and the government bond is considered as default free. Indeed, buying the government bond and selling the corporate bond would be an arbitrage in the frictionless setting but not in the setting with "fixed costs." Hence, the assumption of absence of arbitrage in the frictionless case is not equivalent to the assumption of absence of arbitrage in the case with "fixed costs."

In order to clarify the link between the concepts of arbitrage with "fixed costs" and of frictionless arbitrage, we may remark that we have the following:

Proposition 2. If there is a two-period arbitrage opportunity with "fixed costs," then there exists a portfolio $\theta$ such that

$$
\begin{gathered}
\theta \cdot Z\left(\sigma_{t}\right)<0 \\
\text { and } \theta \cdot Z\left(\sigma_{t+1}\right) \geq 0 \text { for all } \sigma_{t+1} \in f\left(\sigma_{t}\right)
\end{gathered}
$$

for some $t$ and some $\sigma_{t}$ in $\Sigma_{t}$. The converse implication holds also true for "vanishing fixed costs."

The existence of an arbitrage opportunity with "vanishing fixed costs" is equivalent to the existence of a strong form of a frictionless arbitrage, i.e. a portfolio with negative initial cost and nonnegative payoffs.

In order to illustrate this result, consider a two-period binomial model, i.e. a model consisting of one risky security with a value of 1 at date 0 and a value of $u$ in state up and of $d$ in state down at date 1; and one riskless security, with a value of 1 at date 0 and a value of $(1+r)$ in both states of the world at date 1 .

It is easy to see that if $d<(1+r)<u$, then the model is arbitrage-free, in both cases, with and without "fixed costs;" if $d=(1+r)$ or $u=(1+r)$, then it is arbitrage-free in the model with 
"fixed costs" but not in the frictionless model anymore; if $d>(1+r)$ or $u<(1+r)$, then it contains arbitrage opportunities in the frictionless model as well as in the model with "vanishing fixed costs."

A characterization of the absence of arbitrage with "fixed costs" then follows:

Proposition 3. There is no two-period arbitrage with "fixed costs" (resp. "vanishing fixed costs") if (resp. if and only if) for each date $t$ and each date $t$-node $\sigma_{t}$ there exists a nonnegative state-price density on $f\left(\sigma_{t}\right)$ compatible with the asset prices i.e. there exists a family of nonnegative numbers $\left(\pi_{\rho}\right)_{\rho \in f\left(\sigma_{t}\right)}$ such that

$$
Z^{k}\left(\sigma_{t}\right)=\sum_{\sigma_{t+1} \in f\left(\sigma_{t}\right)} \pi_{\sigma_{t+1}} Z^{k}\left(\sigma_{t+1}\right) \quad k=0, \ldots, n
$$

For illustration purpose, let us consider a two-period financial model with one bond and one stock where the riskless rate is equal to the lowest possible rate of return of the stock. According to the Fundamental Theorem of Asset Pricing, since there is an arbitrage opportunity for the frictionless case, there cannot exist a positive compatible state-price density. Nevertheless, the state-price density that puts all the weight on the states of the world such that the riskless rate is equal to the lowest possible rate of return of the stock is compatible with our financial model.

The fact that state price densities can be zero when the physical probability is non zero seems quite striking and counterintuitive. It does not mean that investors have access to units of consumption in these states for free since this access to units of consumption is linked to the payment of a fixed cost in all states. We can wonder whether the situation with zero state price densities in states of positive physical probability is compatible with equilibrium or at least utility maximization. Here again, it does not mean that the agent's marginal utility in these 
states is zero. It just means that the fixed cost structure and the agent's utility function are such that at some point of consumption it is not possible to increase the agent's utility, since any marginal increase of consumption in these states (with zero state price densities) would be compensated by a marginal increase of the fixed cost to pay in all other states. An example of such a situation is provided in the Appendix. Note that this example is not a singular one and we have the following general result. Even if there are zero state-prices, a large class of agents will find a positive optimal solution to a consumption investment problem, namely those agents who have a marginal utility function decreasing faster than the marginal "fixed cost." This condition is for instance satisfied if we assume that the "fixed transaction costs" structure is vanishing but not bounded above and that the utility functions are in the HARA class (i.e. $\left.-\frac{u^{\prime}}{u^{\prime \prime}}(x)=\alpha+\eta x\right)$ and satisfy the empirically reasonable condition $\eta<1$.

The same results can be obtained in a dynamic setting. Furthermore, in such a setting an arbitrage opportunity can be defined as a strategy on the whole tree instead of a strategy between a node and its successors. The next result provides a straightforward extension of the no-arbitrage characterization to such a dynamic framework.

Definition 4. A dynamic arbitrage opportunity is a strategy described by a nonnull dynamic portfolio $\theta$ (i.e. a portfolio $\theta\left(\sigma_{t}\right)$ for all date $t=0, \ldots, T-1$ and all date $t$-node $\sigma_{t}$ ) satisfying for all $t$, for all $\sigma_{t} \in \Sigma_{t}$ and all $\sigma_{t+1} \in f\left(\sigma_{t}\right)$ the following self-financing condition

$$
\theta\left(\sigma_{t}\right) \cdot Z\left(\sigma_{t+1}\right)=\theta\left(\sigma_{t+1}\right) \cdot Z\left(\sigma_{t+1}\right)+c^{\theta}\left(\sigma_{t+1}\right)
$$

and such that

$$
\theta\left(\sigma_{0}\right) \cdot Z\left(\sigma_{0}\right)+c_{0}^{\theta} \leq 0
$$


and, for all $\sigma_{T} \in \Sigma_{T}$,

$$
\begin{aligned}
\theta^{k}\left(\sigma_{T}\right) & =0, \quad k=1, \ldots, n, \\
\theta\left(\sigma_{T}\right) \cdot Z\left(\sigma_{T}\right)-c^{\theta}\left(\sigma_{T}\right) & \geq 0 .
\end{aligned}
$$

The condition $\theta^{k}\left(\sigma_{T}\right)=0, k=1, \ldots, n$ means that the portfolio is liquidated at the final date. We then have:

Proposition 5. The existence of a dynamic arbitrage opportunity is equivalent to the existence of a two period arbitrage opportunity.

As a corollary to Proposition 3, in the presence of "vanishing fixed costs" the no-arbitrage condition implies the existence of a nonnegative compatible state-price density $\pi$ on the whole tree, i.e. $\pi=\left(\pi\left(\sigma_{t}\right)\right)_{t=0, \cdots, T, \sigma_{t} \in \Sigma_{t}}$ such that, at each date $t=0, \cdots, T-1$ and at each date $t$-node $\sigma_{t}$, we have

$$
\pi\left(\sigma_{t}\right) Z\left(\sigma_{t}\right)=\sum_{\sigma_{t+1} \in f\left(\sigma_{t}\right)} \pi\left(\sigma_{t+1}\right) Z\left(\sigma_{t+1}\right) .
$$

Indeed, it suffices to multiply the different state-price densities obtained at each node between that node and its successors to construct a nonnegative state-price density compatible with the assets prices.

Such a state-price density can be seen as a nonnegative (instead of positive as in the frictionless case) subjective probability on the space $\Omega$ of the states of the world and the compatibility condition between the state-price density and the price processes becomes then a martingale condition on the traded assets prices under this subjective probability.

However the existence of a nonnegative compatible state-price density is not a sufficient 
condition to rule out any arbitrage opportunity. We consider the following model for which $T=2, \Omega=\left\{\omega_{1}, \ldots, \omega_{4}\right\}, F_{1}=\left\{\left\{\omega_{1}, \omega_{2}\right\},\left\{\omega_{3}, \omega_{4}\right\}\right\}, n=1$ and the prices are given by:

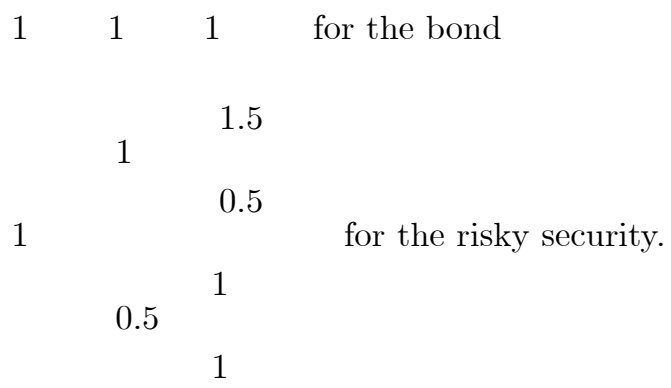

There exists a nonnegative state-price density given by

0.5

1

0.5

1

0

0

Nevertheless, as we have seen earlier, the model is not arbitrage-free: we only need at date 1 and in the lower date 1 -node $\left\{\omega_{3}, \omega_{4}\right\}$ to buy one unit of the risky security and to sell one unit of bond and to clear the position at date 2 to obtain an arbitrage opportunity.

In order to completely characterize the assumption of absence of arbitrage, we need to introduce a whole family of nonnegative state-price densities or equivalently of martingale measures with nonnegative weights. Let us start with some definitions.

For a given date $i \in\{0, \cdots, T-1\}$ and a given date $i$-node $\sigma$, we define the sub-model $S^{\sigma}$ by a set $\Omega^{\sigma}=\sigma$ of states of the world and a family of increasingly finer partitions $\left(F_{t}^{\sigma}\right)_{t \in\{0, \ldots, T-i\}}$ with $F_{t}^{\sigma}=\left\{\sigma^{\prime} \in F_{t+i}: \sigma^{\prime} \subset \sigma\right\}$ and asset prices defined for $\sigma^{\prime} \in F_{t}^{\sigma}$, for $t$ in $\{0, \ldots, T-i\}$, by $Z^{\sigma}\left(\sigma^{\prime}\right)=Z\left(\sigma^{\prime}\right)$. In fact, the sub-model $S^{\sigma}$ can be described by the sub-tree starting in $\sigma$. 
Theorem 6. There is no dynamic arbitrage opportunity with "vanishing fixed costs" (resp. "fixed costs") if and only if (resp. if) for all date $i$ and all date $i$-node $\sigma$, there exists a nonnegative state-price density $\pi^{\sigma}=\left(\pi^{\sigma}\left(\sigma_{t}\right)\right)_{t=0, \cdots, T-i, \sigma_{t} \in F_{t}^{\sigma}}$ compatible with the asset prices in the sub-model $S^{\sigma}$, i.e.for all $t \in\{0, \cdots, T-i\}$ and all $\sigma_{t} \in F_{t}^{\sigma}$

$$
\pi^{\sigma}\left(\sigma_{t}\right) Z^{\sigma}\left(\sigma_{t}\right)=\sum_{\sigma_{t+1} \in f\left(\sigma_{t}\right)} \pi^{\sigma}\left(\sigma_{t+1}\right) Z^{\sigma}\left(\sigma_{t+1}\right)
$$

The absence of arbitrage opportunities is then characterized by the existence of martingale measures with nonnegative weights for every sub-tree.

\section{B. Pricing in Models with "Fixed Costs"}

In this subsection, we restrict our attention to "vanishing fixed costs structures."

A contingent claim $x$ is defined by its payoffs in each terminal node $\sigma_{T} \in \Sigma_{T}$. It pays, at date $T$, the amount $x\left(\sigma_{T}\right)$ at the node $\sigma_{T}$.

A contingent claim $x$ is said to be attainable (in the frictionless model) if there exists some dynamic portfolio $\theta$ satisfying for all $t$, for all $\sigma_{t} \in \Sigma_{t}$ and all $\sigma_{t+1} \in f\left(\sigma_{t}\right)$ the self-financing frictionless condition $\theta\left(\sigma_{t}\right) \cdot Z\left(\sigma_{t+1}\right)=\theta\left(\sigma_{t+1}\right) \cdot Z\left(\sigma_{t+1}\right)$ such that $\theta\left(\sigma_{T}\right) \cdot Z\left(\sigma_{T}\right)=x$ for all $\sigma_{T} \in \Sigma_{T}$. Let $M$ denote the set of all attainable contingent claims. Note that $M$ is a linear space.

Definition 7. A fair pricing rule on the set of attainable claims $M$ is a functional $p$ defined on $M$, such that

1. $p$ induces no arbitrage, i.e. it is not possible to find a dynamic portfolio $\theta$ satisfying the self-financing condition $\theta\left(\sigma_{t}\right) \cdot Z\left(\sigma_{t+1}\right)=\theta\left(\sigma_{t+1}\right) \cdot Z\left(\sigma_{t+1}\right)+c^{\theta}\left(\sigma_{t+1}\right)$ and contingent claims 
$m_{1}, \ldots, m_{d}$ in $M$ for which $\sum_{i=1}^{d} p\left(m_{i}\right)+\theta\left(\sigma_{0}\right) \cdot Z\left(\sigma_{0}\right)+c_{0}^{\theta} \leq 0, \sum_{i=1}^{d} m_{i}+\theta \cdot Z-c_{T}^{\theta} \geq 0$ on $\Sigma_{T}$ and one of the two is nonnull.

2. $p(m) \leq p_{s}(m)$, where

$$
p_{s}(m) \equiv \inf \left\{\theta\left(\sigma_{0}\right) \cdot Z\left(\sigma_{0}\right)+c_{0}^{\theta}, \theta \text { is self financing and } \theta \cdot Z-c_{T}^{\theta} \geq m \text { on } \Sigma_{T}\right\}
$$

Part 1 is the usual no-arbitrage condition; it encompasses the fact that the model is arbitragefree and could be replaced in a frictionless model by $\sum_{i=1}^{d} p\left(m_{i}\right) \leq 0, \sum_{i=1}^{d} m_{i} \geq 0$ and one of the two is nonnull. Part 2 says that a fair price for $m$ is smaller than its superreplication price and corresponds to a monotonicity assumption; we impose that if it is possible to obtain a better payoff than $m$ at a cost $p_{s}(m)$, then no one will accept to pay more than $p_{s}(m)$. Note that since $m$ is attainable by a frictionless self financing strategy, and since the total cost incurred for any strategy is bounded, there always exists at least a self financing (inclusive of transaction costs) strategy dominating $m$.

The following proposition gives us the fair pricing rules on $M$ through the use of the nonnegative state-price densities on the whole tree $\pi^{\Omega}$ introduced in Theorem 6 . Notice that the linear functional $l$ given by $l\left[\left(\theta\left(\sigma_{T}\right) \cdot Z\left(\sigma_{T}\right)\right)_{\sigma_{T} \in \Sigma_{T}}\right]=\theta\left(\sigma_{0}\right) \cdot Z\left(\sigma_{0}\right)$ for all frictionless self financing strategy $\theta$ is well defined on $M$ : indeed, if two frictionless self financing strategies $\theta$ and $\theta^{\prime}$ generate the same contingent claim $m$, i.e. if their terminal values are both equal to $m$, then their initial value must also be equal; if not, the strategy given by $\theta-\theta^{\prime}$ or by $\theta^{\prime}-\theta$ would be a strong form of frictionless arbitrage opportunity, which would lead to the existence of an arbitrage opportunity with "vanishing fixed costs" as seen in Proposition 1. We find that any fair pricing rule can be written as the sum of the linear functional $l$ and of a "vanishing 
fixed cost," or expressed in terms of the nonnegative state-price densities found in the preceding section,

Proposition 8. If the "fixed costs structure" is bounded (resp. vanishing), then any fair pricing rule $p$ on the set of attainable claims $M$ is given by

$$
p(m)=\sum_{\sigma_{T} \in \Sigma_{T}} \pi^{\Omega}\left(\sigma_{T}\right) m\left(\sigma_{T}\right)+c(m) \quad \text { for all } m \text { in } M
$$

where $\pi^{\Omega}$ is any nonnegative state-price density as determined in Theorem 6 and where $c$ is bounded (resp. vanishing i.e. $\left.\frac{c(\lambda m)}{\lambda} \rightarrow_{\lambda \rightarrow \infty} 0\right)$.

Moreover, if $p(\lambda x) \leq \lambda[p(x)]$, for $\lambda$ sufficiently large, then the "fixed cost" function $c(\cdot)$ is nonnegative.

This implies that $\frac{p(\lambda m)}{\lambda} \rightarrow_{\lambda \rightarrow \infty} \sum_{\sigma_{T} \in \Sigma_{T}} \pi^{\Omega}\left(\sigma_{T}\right) m\left(\sigma_{T}\right)$ for any attainable contingent claim $m$. Therefore, the unit price of any attainable contingent claim $m$ is given by $\sum_{\sigma_{T} \in \Sigma_{T}} \pi^{\Omega}\left(\sigma_{T}\right) m\left(\sigma_{T}\right)$ in the limit of large quantities traded.

As usual, we say that the market is complete (in the frictionless model) if any contingent claim is attainable (in the frictionless model). It is then easy to see that if the market is arbitrage-free and complete, there exists a unique nonnegative state-price density and there exists a unique fair price for any contingent claim in the limit of large quantities.

If we further assume that the frictionless model is arbitrage-free, the unique state-price density is then positive and the unique fair price for a contingent claim in the model with "vanishing fixed costs" is equal to the frictionless price in the limit of large quantities. If the market is incomplete, the set of possible prices ${ }^{5}$ in the model with "vanishing fixed costs" for a

\footnotetext{
${ }^{5}$ The set of possible prices is defined, as usual, as the set of prices given by all the possible fair pricing rules.
} 
contingent claim is equal to the set of possible frictionless prices (and their limits).

\section{Applications}

In this section, we apply the results of Section 2 in order to show that popular term structure of interest rate models that are rejected by the results in Dybvig, Ingersoll and Ross (1996) are indeed arbitrage-free in the presence of "fixed costs" (including arbitrarily small ones). We also show that "fixed costs" allow us to use models where asset prices are bounded and reach their bound. In particular, we obtain yield curve shapes as in Longstaff (1992), in a Cox, Ingersoll and Ross (1985) like model but without assuming an absorbing barrier for the short rate. As in Section 2, all the models under consideration are in a discrete time framework. This permits to provide simple intuitions about how things work. It would be possible to generalize them in continuous time through some technical adjustment. For instance, in order to construct arbitrage free models where asset prices are bounded and reach their bound it suffices to consider processes that have a positive probability to be absorbed when they reach the bound. The absolutely continuous state price density will then put a zero-weight on all the trajectories that are not absorbed and the whole weight on the absorbed trajectories. The same kind of considerations apply in the Dybvig, Ingersoll and Ross (1996) property discussion: it suffices to consider interest rate models where the nondecreasing trajectories have a positive weight under the initial probability and to put all the weight on these trajectories in the state price density.

\section{A. Long Rates can Fall}

We start by proving that the quite striking result obtained by Dybvig, Ingersoll and Ross (1996), which asserts that, under the assumption of absence of arbitrage, long-term zero-coupon rates can never fall, is not true in models with "fixed costs." We then show that in Ho and Lee 
(1986)-like term structure interest rate models, long-term rates can fluctuate if there are "fixed costs." Finally, we show that, with "fixed costs," the Brennan and Schwartz (1979) model is indeed arbitrage-free.

\section{A Simple Example}

Let us recall the framework of Dybvig, Ingersoll and Ross (1996). There are traded zero coupon bonds of all integer maturities. For $t<t^{\prime}$, we define $\nu\left(t, t^{\prime}\right)$ to be the discount bond price, i.e. the price at $t$ of receiving a unit payoff at some later date $t^{\prime}$. The zero-coupon rate $z\left(t, t^{\prime}\right)$ is defined implicitly by

$$
\nu\left(t, t^{\prime}\right) \equiv \frac{1}{\left[1+z\left(t, t^{\prime}\right)\right]^{t^{\prime}-t}}
$$

The long-term zero-coupon rate at time $t$, is given by $z_{L}(t)=\lim _{t^{\prime} \rightarrow \infty} z\left(t, t^{\prime}\right)$ if the limit exists.

A free lunch is defined as a sequence of net trades such that $i$ ) the payoff tends uniformly to a nonnegative random variable that is positive with positive probability and the price tends to zero or $i i$ ) the price tends to a negative number but the payoff tends uniformly to a nonnegative random variable.

Then the authors prove that, under the assumption of no-free lunch, if for $s<t$, the long-term zero coupon rate exists at time $s$ and exists with probability one at time $t$, then $z_{L}(s) \leq z_{L}(t, \omega)$ with probability one (Dybvig, Ingersoll and Ross, 1996, Theorem 2, p.9). To do so, they use an arbitrage argument of the form $i$ ). However, if we transpose their result in our discrete time and finite states of the world framework, the set of attainable payoffs is closed and a free-lunch is in fact an arbitrage.

Their result can then be rephrased in our framework as follows. An arbitrage is a net trade 
with zero cost and nonnegative, nonzero terminal payoff. Under the assumption of no-arbitrage, if for $s<t$, the long-term zero coupon rate exists at time $s$ and exists with probability one at time $t$, then $z_{L}(s) \leq z_{L}(t, \omega)$ with probability one.

As a consequence of Dybvig, Ingersoll and Ross (1996) result, models where the long-term rate follows a specified nonmonotone behavior and in particular models where the long-term rate follows the discrete time analogon of a diffusion are rejected by the no-arbitrage condition. However this does not mean that models where the long-term yield is a risk factor as in Duffie and Kan (1996) have to be systematically rejected. Indeed, in these models, the long-term yield corresponds to a yield associated to a long but finite horizon while in Dybvig, Ingersoll and Ross (1996), the long-term rate is the limit rate when the horizon goes to infinity. In fact, it is possible to construct models where

- for any $t$, the function $t^{\prime} \rightarrow z\left(t, t^{\prime}\right)$ is nonmonotone,

- for any $T$, the function $t \rightarrow z(t, t+T)$ is nonmonotone,

- however, $t \rightarrow z_{L}(t)$ have a monotone nondecreasing behavior.

Such an example is provided in the Appendix.

We show on a simple counterexample that in models with "fixed costs," long-term zerocoupon rates can fall. Suppose it is known that the spot rate will be $r_{1}$ until $s$, and that at time $s$ it will be revealed to either shift to $r_{2}\left(<r_{1}\right)$ forever or to remain at $r_{1}$ forever, which is illustrated by

$$
\begin{aligned}
1 \rightarrow \ldots \rightarrow\left(1+r_{1}\right)^{s} & \rightarrow\left(1+r_{1}\right)^{s+1} \quad \rightarrow \ldots \rightarrow\left(1+r_{1}\right)^{t} \quad \rightarrow \ldots \\
& \searrow\left(1+r_{1}\right)^{s}\left(1+r_{2}\right) \rightarrow \ldots \rightarrow\left(1+r_{1}\right)^{s}\left(1+r_{2}\right)^{t-s} \rightarrow \ldots
\end{aligned}
$$


We assume that the discount bond prices are given (for $t^{\prime} \geq t$ ) by $\nu\left(t, t^{\prime}\right)=\frac{1}{\left(1+r_{1}\right)^{t^{\prime}-t}}$ for $t \leq s$ and, for $t>s$, by $\nu\left(t, t^{\prime}\right)=\frac{1}{\left(1+r_{1}\right)^{t^{\prime}-t}}\left(\right.$ resp. $\left.\frac{1}{\left(1+r_{2}\right)^{t^{\prime}-t}}\right)$ if $r_{1}$ (resp. if $r_{2}$ ).

In fact, for $t^{\prime} \geq t>s$ and for $s \geq t^{\prime} \geq t$, interest rates are deterministic and bond prices are uniquely determined without making any assumption on the state-price density or on the risk premium. For $t^{\prime}>s \geq t$, our bond prices correspond to a state-price density which puts a zero weight on the lower branch of the tree. In a "fixed costs" framework, by Theorem 6 , this model does not contain any arbitrage opportunity in a finite horizon. As far as free lunches are concerned, it is clear that we need not consider strategies starting after date $s+1$. For strategies starting before date $s$, it is easy to see that any net trade in the state $r_{1}$ is equal to 0 if we do not take the "fixed costs" into account and is smaller than $-\mathbf{c}$ if we take them into account. Hence, there is no sequence of net trades, taking the "fixed costs" into account, converging to a nonnegative nonnull random variable.

We then obtain $z_{L}(t)=r_{1}$ for $t \leq s$ and for $t>s, z_{L}(t)=r_{1}$ if $r_{1}$ and $z_{L}(t)=r_{2}$ if $r_{2}$, so that, since $r_{2}<r_{1}$, we do not have $z_{L}(s) \leq \min _{\omega} z_{L}(t, \omega)$ (more precisely, for all $t_{1} \leq s$ and all $t_{2}>s$, we have $z_{L}\left(t_{1}\right) \geq z_{L}\left(t_{2}, \omega\right)$ with probability one and $\left.P\left[z_{L}\left(t_{1}\right)>z_{L}\left(t_{2}, \omega\right)\right]>0\right)$.

We have then proved that when there are "fixed costs" (including arbitrarily small ones), the result in Dybvig, Ingersoll and Ross (1996) is no longer true : long-term rates can fall. In our example they fall with a probability that can be chosen arbitrarily, namely the probability for the short-term rate to shift from $r_{1}$ to $r_{2}$ at date $s$.

\section{Ho and Lee-like Models}

It is shown in Dybvig, Ingersoll and Ross (1996, Section $I V$ ), that in the Ho and Lee (1986) model, the long-term rates can only increase. We prove here that with the introduction of "fixed costs," long-term rates can decrease in Ho and Lee-like models. More precisely, we first give an 
example of an arbitrage-free Ho and Lee model in which long-term rates fall with probability one. We then give an example of a trinomial model, analogous to Ho and Lee, for which longterm rates can both increase or decrease by introducing "fixed costs" (even arbitrarily small ones).

Suppose that $\Sigma_{t}=\{u, d\}^{t}$. In such a context, a date- $t$ node $\sigma_{t}$ is a sequence $\left(\rho_{1}, \cdots, \rho_{t}\right)$ of elements of $\{u, d\}$ and, for $\sigma_{t}=\left(\rho_{1}, \cdots, \rho_{t}\right), f\left(\sigma_{t}\right)=\left\{\left(\sigma_{t}, u\right),\left(\sigma_{t}, d\right)\right\}$ with, by definition, $\left(\sigma_{t}, u\right)=\left(\rho_{1}, \cdots, \rho_{t}, u\right)$ and $\left(\sigma_{t}, d\right)=\left(\rho_{1}, \cdots, \rho_{t}, d\right)$.

Assume now as in Ho and Lee (1986) that the value of any portfolio is independent from the chosen path and that discount bond prices satisfy:

$$
\nu\left(t, T,\left(\sigma_{t-1}, \rho_{t}\right)\right)=\frac{\nu\left(t-1, T, \sigma_{t-1}\right)}{\nu\left(t-1, t, \sigma_{t-1}\right)} H\left(T-t, \rho_{t}\right)
$$

with $H(\cdot, \rho)=h(\cdot)$, if $\rho=u, H(\cdot, \rho)=h^{*}(\cdot)$, if $\rho=d$, and $h \neq h^{*}$.

Proposition 9. If there are "fixed costs," the functions $h$ and $h^{*}$ can be chosen such that $h$ is constant equal to 1 and $h^{*}(n)=\left[h^{*}(1)\right]^{n}>1$ and the model is then arbitrage-free. Furthermore, in such a framework we have

$$
\begin{aligned}
& 1+z_{L}\left(t,\left(\sigma_{t-1}, u\right)\right)=1+z_{L}\left(t-1, \sigma_{t-1}\right) \\
& 1+z_{L}\left(t,\left(\sigma_{t-1}, d\right)\right)<1+z_{L}\left(t-1, \sigma_{t-1}\right)
\end{aligned}
$$

Hence we have proved that the Ho and Lee (1986) model can lead to nonincreasing long-term rates in the presence of "fixed costs" including arbitrarily small ones. In fact, in our setting the long-term rates fall with a probability equal to one.

In order to construct a model where the long-term rates can fluctuate (i.e. increase and 
decrease depending on the states of the world), we have to extend the Ho and Lee (1986) model to a trinomial setting.

In order to construct such a trinomial generalization of the Ho and Lee (1986) model, we still assume that the value of any portfolio is independent from the chosen path and that

$$
\nu\left(t, T,\left(\sigma_{t-1}, \rho_{t}\right)\right)=\frac{\nu\left(t-1, T, \sigma_{t-1}\right)}{\nu\left(t-1, t, \sigma_{t-1}\right)} H\left(T-t, \rho_{t}\right)
$$

but with $\Sigma_{t}=\{u, m, d\}^{t}$. In the next, such a model is called a generalized trinomial Ho and Lee model. We then have the following result

Proposition 10. In a "fixed costs" framework, there exists an arbitrage-free specification of the generalized trinomial Ho and Lee model such that

$$
\begin{aligned}
& 1+z_{L}\left(t,\left(\sigma_{t-1}, u\right)\right)>1+z_{L}\left(t-1, \sigma_{t-1}\right) \\
& 1+z_{L}\left(t,\left(\sigma_{t-1}, m\right)\right)=1+z_{L}\left(t-1, \sigma_{t-1}\right) \\
& 1+z_{L}\left(t,\left(\sigma_{t-1}, d\right)\right)<1+z_{L}\left(t-1, \sigma_{t-1}\right)
\end{aligned}
$$

Hence we have constructed a Ho and Lee like model where long-term rates can fluctuate. More precisely they increase along the upper branches of the trinomial tree and they decrease along the lower branches. Consequently, models where long-term rates have a nonmonotonic behaviour are no longer rejected for arbitrage considerations. ${ }^{6}$

\footnotetext{
${ }^{6}$ As underlined by Dybvig, Ingersoll and Ross (1996) in a frictionless framework, "It is not permissible to specify a term structure model with a stochastic factor which is the long (asymptotic) end of the zero-coupon yield curve unless this factor can only increase over time".
} 


\section{The Brennan and Schwartz Model}

In the Brennan and Schwartz (1979) model the long-term rate is assumed to follow a diffusion process and according to Dybvig, Ingersoll and Ross (1996) is not compatible with the noarbitrage condition. In this section we consider a discrete time version of Brennan and Schwartz model and we prove that such a model where the long-term rates follow a diffusion process (or more precisely a discrete time approximation of a diffusion process) is compatible with the no arbitrage condition in the presence of "fixed costs," even arbitrarily small. Consequently, even if in such a model the long-term rates fluctuate and decrease in some states of the world, we cannot reject it based on arbitrage considerations if there are "fixed costs" (including arbitrarily small ones). This enables to reconcile arbitrage conditions and the empirical observations that do not seem to reject Brennan and Schwartz (1979) model (see Chan et al., 1992).

Let us first recall that in the Brennan and Schwartz (1979) model, the short-term rate and the long-term rate, respectively denoted by $\ell$ and $r$, are governed by the following diffusion equation

$$
\left\{\begin{array}{l}
d \ell_{t}=\mu\left(\ell_{t}, r_{t}, t\right) d t+\sigma_{1} \ell_{t} d W_{t}^{1} \\
d r_{t}=a\left(\ell_{t}-r_{t}\right) d t+\sigma_{2} \sqrt{r_{t}} d W_{t}^{2}
\end{array}\right.
$$

where $a, \sigma_{1}$ and $\sigma_{2}$ are given positive constants and where $\mu$ is a given bounded function of the current date and of the long-term and short-term rates.

In the next we consider the following model for the long-term and short-term rates. It is not recombining but our aim is to show the acceptability of such a model and as we will see, we obtain simple pricing formulas. Let 


$$
\begin{aligned}
& \ell_{n+1}=\ell_{n} \begin{cases}\exp \left\{\left(\frac{\mu\left(\ell_{n}, r_{n}, n \frac{T}{N}\right)}{\ell_{n}}-\frac{1}{2} \tilde{\sigma}_{1}^{2}\right) \frac{T}{N}+\tilde{\sigma}_{1} \sqrt{\frac{T}{N}}\right\} & \text { with a probability } p_{u} \\
1 & \text { with a probability } p_{m} \\
\exp \left\{\left(\frac{\mu\left(\ell_{n}, r_{n}, n \frac{T}{N}\right)}{\ell_{n}}-\frac{1}{2} \tilde{\sigma}_{1}^{2}\right) \frac{T}{N}-\tilde{\sigma}_{1} \sqrt{\frac{T}{N}}\right\} & \text { with a probability } p_{d}\end{cases} \\
& r_{n+1}=r_{n} \begin{cases}\exp \left\{\left(\frac{a\left(\ell_{n}-r_{n}\right)}{r_{n}}-\frac{1}{2} \frac{\sigma_{2}^{2}}{r_{n}}\right) \frac{T}{N}+\sqrt{2} \frac{\sigma_{2}}{\sqrt{r_{n}}} \sqrt{\frac{T}{N}}\right\} & \text { with a probability } q_{u} \\
\exp \left\{\left(\frac{a\left(\ell_{n}-r_{n}\right)}{r_{n}}-\frac{1}{2} \frac{\sigma_{2}^{2}}{r_{n}}\right) \frac{T}{N}\right\} & \text { with a probability } q_{m} \\
\exp \left\{\left(\frac{a\left(\ell_{n}-r_{n}\right)}{r_{n}}-\frac{1}{2} \frac{\sigma_{2}^{2}}{r_{n}}\right) \frac{T}{N}-\sqrt{2} \frac{\sigma_{2}}{\sqrt{r_{n}}} \sqrt{\frac{T}{N}}\right\} & \text { with a probability } q_{d}\end{cases}
\end{aligned}
$$

where $T$ is a given horizon, $\tilde{\sigma}_{1}, a, \sigma_{2}$ are positive constants and where $\mu$ is a given bounded function.

This model consists of 9 different states of the world at each date, with a probability $p_{i} q_{j}$ for each state $(i, j)_{\substack{i=u, m, d \\ j=u, m, d}}$ and we have the following result

Proposition 11. There exists a specification of the probabilities $\left(p_{u}, p_{m}, p_{d}\right)$ and $\left(q_{u}, q_{m}, q_{d}\right)$ such that the model above is a discrete time approximation of the Brennan and Schwartz (1979) model. Furthermore, in a "fixed costs" setting, there exists an arbitrage-free bond price structure compatible with this model, i.e. such that the short-term rate is given by $r$ and the long-term rate by $\ell$.

Hence we have proved that in the presence of "fixed costs," the Brennan and Schwartz (1979) model (or more precisely a discrete time approximation of this model) is compatible with the no-arbitrage condition. This compatibility is due to the introdution of "fixed costs" since in such a model the long-term rates fluctuate and following Dybvig, Ingersoll and Ross (1996) this property leads to arbitrage opportunities in a frictionless setting. There obviously might be many other frictions that might lead to the same result; however the introduction of "fixed 
costs" is the only one ${ }^{7}$ for which arbitrage opportunities vanish and such that by Proposition 8 the bond prices structure remains the same as in the frictionless setting (at least in the limit of large quantities).

\section{B. Price Processes with Upper and Lower Bounds or Absorbing Barriers}

We now turn to securities price models that are bounded and can potentially reach their bounds. We then propose a discrete-time version of a Cox, Ingersoll and Ross (1985) model which, as in Longstaff (1992), produces yield curves with realistic humps, but without assuming an absorbing barrier for the short rate by introducing "fixed costs."

\section{The Binomial Model of Cox, Ross and Rubinstein (1979) with Bounds}

The introduction of "fixed costs" enables us to consider as arbitrage-free models where the asset price processes reach some bounds and to determine in such a framework a fair pricing rule for contingent claims. Indeed if we assume that the discounted price process of a given asset is bounded by an upper barrier and reaches this barrier without being absorbed then it is clear that there is no positive state-price density compatible with this asset price process. This price process leads then to arbitrage opportunities in a frictionless setting. However a state-price density which, once the barrier reached, puts all the weight on the constant discounted price path might be compatible with this asset price process. By Theorem 6 the presence of upper bounds on the discounted asset prices may then be compatible with the no-arbitrage condition in a "fixed costs" setting.

We will illustrate this property on a version of the binomial model of Cox, Ross and Ru-

\footnotetext{
${ }^{7}$ Among the classical frictions studied in the litterature (proportional transaction costs, shortselling costs, portfolio constraints,...)
} 
binstein (1979) with bounds. This model has been studied in Sondermann (1988), ${ }^{8}$ for other purposes.

More precisely, denote by $\left(W_{t}\right)_{t \in\{0, \cdots, T\}}$ the stochastic process given by:

$$
W_{t}=Z_{0} \cdot \prod_{n=1}^{t}\left(1+\rho_{n}\right)
$$

where $\left(\rho_{n}\right)$ is a family of independent random variables such that

$$
\rho_{n}=\left\{\begin{array}{cc}
\delta+\frac{1}{2} \delta^{2} \stackrel{\text { def }}{=} \delta_{u} & \text { with probability } p \\
-\delta+\frac{1}{2} \delta^{2} \stackrel{\text { def }}{=} \delta_{d} & \text { with probability } 1-p
\end{array}\right.
$$

for fixed $2>\delta>0,0<p<1$. The process $\left(W_{t}\right)_{t \in\{0, \cdots, T\}}$ is a geometric random walk with compensated stochastic drift and corresponds to the security price process in the Cox, Ross and Rubinstein model (1979). Note that in such a context, a date- $t$ node $\sigma_{t}$ is a sequence $\left(\rho_{1}, \cdots, \rho_{t}\right)$. As we have mentioned, we assume that for some reason, the price process cannot go beyond some a priori given bounds. We shall here fix an upper bound $M$ and we assume for simplicity of exposition that $M$ belongs to the set of values taken by the process $\left(W_{t}\right)_{t \in\{0, \cdots, T\}}$. The price process of our security is given by $\left(Z_{t}\right)_{t \in\{0, \cdots, T\}}$ with $Z_{0}=W_{0}$ and where, for $\sigma_{t}=$ $\left(\rho_{1}, \cdots, \rho_{t}\right)$ and $\sigma_{t+1}=\left(\rho_{1}, \cdots, \rho_{t+1}\right) \in f\left(\sigma_{t}\right)$,

$$
\begin{aligned}
& Z\left(\sigma_{t+1}\right)=Z\left(\sigma_{t}\right)\left(1+\rho_{t+1}\right) \quad \text { if } Z\left(\sigma_{t}\right)<M \text { and, if } Z\left(\sigma_{t}\right)=M, \\
& M \quad \text { if } \rho_{t+1}=\delta_{u} \\
& Z\left(\sigma_{t+1}\right)=\left\{\begin{array}{c} 
\\
Z\left(\sigma_{t}\right)\left(1+\rho_{t+1}\right) \quad \text { if } \rho_{t+1}=\delta_{d}
\end{array}\right.
\end{aligned}
$$

\footnotetext{
${ }^{8}$ This paper might be difficult to consult. See also Sondermann (1987).
} 
In the next this model is called the generalized Cox, Ross and Rubinstein model (1979) with bounds. The aim is to value a European call $x=\left(Z_{T}-K\right)_{+}$for some a priori given bound $M \geq Z_{0}$.

We have the following result

Proposition 12. The generalized Cox, Ross and Rubinstein model (1979) with bounds is arbitrage-free in a "fixed costs" setting. Furthermore, if the "fixed costs structure" is vanishing, the price of a European call option in this model is equal (in the limit of large quantities) to the price of a barrier option with same strike and maturity in the frictionless Cox, Ross and Rubinstein model (1979).

It is shown in Sondermann (1988) that the corresponding option pricing formula can be simplified for small grid sizes. Letting the grid size go to zero, by the Central Limit Theorem the analog of the Black and Scholes (1973) formula is obtained for such options and then for European call options with bounds on the underlying asset. These formulas reduce to the Black and Scholes formula if the upper bound goes to infinity. The same kind of results can be obtained if we introduce a lower bound on the asset prices and the pricing formulas we obtain reduce to the Black and Scholes formula if the lower bound approaches zero.

Hence we have proved that the presence of "fixed costs" (including arbitrarily small ones) allows us to use models with bounds on the discounted asset price processes. Such bounds might be subjectively assumed by some investors in order to model the fact that, from their point of view, the price of a given asset cannot exceed a given level. They can also be seen as objective bounds due to policy constraints as in target zones where some bounds are imposed on the exchange rates (as in the previous European Monetary System). They finally can be seen as 
objective bounds resulting from the choice of a given model.

Such situations can be encountered for instance in interest rate models where $r=0$ is an absorbing barrier as in Longstaff (1992). Indeed, in such models, the price of a given bond when the barrier $r=0$ is reached is equal to 1 which corresponds to an upper bound for a bond price in models where the short-term rates are always nonnegative. Longstaff (1992) introduced such models in order to enlarge the set of possible shapes of the interest rate term structure in a Cox, Ingersoll and Ross model (1985).

\section{The CIR Model with Partially Absorbing Barrier}

The Cox, Ingersoll and Ross (1985) model has been empirically tested by Brown and Dybvig (1986) and by Brown and Schaefer (1994). These authors have shown that the volatility of the short-term rate conforms relatively well to empirical observations, but they argue that the model is not flexible enough to capture the variety of shapes encoutered in practice. More precisely, while this model allows for inverted shapes at the short end of the curve, it fails at capturing yield curves with a hump. Longstaff (1992) proposes an extension of this model allowing for an absorbing barrier at $r=0$ for the short-term rate. He provides then analytical pricing formulas for the bonds and argues that this model leads to more realistic term structures of interest rates. The aim of this section is to show that Longstaff (1992)'s approach and formulas (or more precisely their discrete time version) are compatible with the no arbitrage condition with "fixed costs," even if $r=0$ is not an absorbing barrier. This permits to enlarge the set of possible shapes of the interest rate term structure, and as underlined by Longstaff (1992) to generate possible humps, without imposing the unrealistic absorbing barrier condition. Indeed, it is difficult to accept that when the short-term rate reaches zero it will stay at that level forever.

Let us consider the following model for the short-term rate 


$$
r_{n+1}=r_{n}+\left\{\begin{array}{lr}
a\left(\ell-r_{n}\right) \frac{T}{N}+\sqrt{2} \sigma \sqrt{r_{n}} \sqrt{\frac{T}{N}} & \text { with a probability } 1 / 4 \\
a\left(\ell-r_{n}\right) \frac{T}{N} & \text { with a probability } 1 / 2 \\
a\left(\ell-r_{n}\right) \frac{T}{N}-\sqrt{2} \sigma \sqrt{r_{n}} \sqrt{\frac{T}{N}} & \text { with a probability } 1 / 4
\end{array}\right.
$$

Following Nelson and Ramaswamy (1990) this model is a discrete time approximation of the Cox, Ingersoll and Ross model (1985) model (CIR) for which the short-term rate is governed by the following diffusion equation

$$
d r_{t}=a\left(\ell-r_{t}\right) d t+\sigma \sqrt{r_{t}} d W_{t}
$$

For this reason, the discrete time model introduced above is referred to in the next as the discrete CIR model.

If $2 \sigma^{2}<a \ell$ as imposed by Cox, Ingersoll and Ross model (1985) and if $r_{0}>0$ then both models lead to a (strictly) positive short-term rate at any date with probability one. The Cox, Ingersoll and Ross model (1985) does not explore the case where the condition $2 \sigma^{2}<a \ell$ is not satisfied and where the short-term rate might be equal to zero with a positive probability.

However, Longstaff (1992) pointed out that this condition can be relaxed if we impose a boundary condition on the bond prices when the short-term rate is equal to zero. In particular, he considers an absorbing barrier at $r=0$ for the short-term rate and imposes bond prices equal to one once the barrier is reached. In that case, he provides analytical pricing formulas for the bonds. 
The discrete time version of Longstaff (1992) absorbing barrier model is given by :

$$
\begin{aligned}
& r_{n+1}=r_{n}+ \begin{cases}a\left(\ell-r_{n}\right) \frac{T}{N}+\sqrt{2} \sigma \sqrt{r_{n}} \sqrt{\frac{T}{N}} & 1 / 4 \\
a\left(\ell-r_{n}\right) \frac{T}{N} & 1 / 2 \text { if } r_{n} \neq 0 \\
{\left[a\left(\ell-r_{n}\right) \frac{T}{N}-\sqrt{2} \sigma \sqrt{r_{n}} \sqrt{\frac{T}{N}}\right]_{+}} & 1 / 4\end{cases} \\
& r_{n+1}=r_{n} \text { if } r_{n}=0 .
\end{aligned}
$$

In the next the discrete time version of the bond prices formulas obtained by Longstaff (1992) in the absorbing barrier framework will be called Longstaff's bond prices.

Let us modify the discrete CIR model as follows.

$$
r_{n+1}=r_{n}+\left\{\begin{array}{lc}
a\left(\ell-r_{n}\right) \frac{T}{N}+\sqrt{2} \sigma \sqrt{r_{n}} \sqrt{\frac{T}{N}} & 1 / 4 \\
a\left(\ell-r_{n}\right) \frac{T}{N} & 1 / 2 \\
{\left[\varphi\left(r_{n}\right)\right]_{+}} & 1 / 4
\end{array}\right.
$$

where

$$
\varphi\left(r_{n}\right)= \begin{cases}a\left(\ell-r_{n}\right) \frac{T}{N}-\sqrt{2} \sigma \sqrt{r_{n}} \sqrt{\frac{T}{N}} & \text { if } r_{n}>0 \\ 0 & \text { if } r_{n}=0\end{cases}
$$

This model corresponds to a generalization of the discrete CIR model with a partially absorbing barrier at $r=0$. Indeed, when $r_{n}$ is positive, we define $r_{n+1}$ as in the discrete CIR model with only one modification : $a\left(\ell-r_{n}\right) \frac{T}{N}-\sqrt{2} \sigma \sqrt{r_{n}} \sqrt{\frac{T}{N}}$ in the lower branch is replaced by its positive part in order to ensure that the short-term rate remains positive. When $r_{n}$ is equal to zero, then there are three possible states of the world at date $n+1$; for one of them the short-term rate is absorbed and remains equal to zero and for the two others the short-term rate is reflected. In the next we refer to this model as the partially absorbing CIR model. 
Proposition 13. For $\lambda$ small enough and $N$ large enough (more precisely for $|\lambda|<\frac{a}{\sqrt{2}}$ and $\left.\lambda^{2} \frac{T}{N} \leq \sigma^{2} \inf \left\{\frac{1}{r_{0}},\left(\frac{2 a h}{\sqrt{2} \sigma+\sqrt{2 \sigma^{2}+4 a^{2} \ell h^{2}}}\right)^{2}\right\}\right)$, Longstaff's bond prices are compatible with the partially absorbing CIR model with "fixed costs" (in the sense that these prices can be derived in an arbitrage-free way from the partially absorbing model for the short-term rate).

In order to obtain the same term structure as in the absorbing barrier model we choose a risk-neutral probability for which zero is actually an absorbing barrier. This is made possible by the fact that in the presence of "fixed costs" the risk-neutral probability and the actual probability do not have to be equivalent. A given barrier can then be absorbing under one of them only.

Our term structure is then the discrete time analog of the specific example of Longstaff (1992, Section 3) and allows for humps at longer maturities. However, the short-term rate dynamics is not the same: we do not assume that zero is an absorbing barrier. We only impose that when the short-term rate reaches zero, there is a nonnull probability that it stays at zero, for at least one period. As underlined by Longstaff (1992), the behavior of Treasury-bill yields appears to be more consistent with such a sticky boundary than with a totally reflecting boundary: extremely low levels of yields tend to persist rather than immediately increasing back toward higher levels.

\section{Conclusion}

We have shown that with the introduction of "fixed costs" (including arbitrarily small ones), we can enlarge the set of possible arbitrage-free models to include popular models of the term structure of interest rates. In particular, models with bounded prices processes reaching their bounds are now arbitrage-free. In addition, we have relaxed the monotonicity constraint imposed 
for arbitrage considerations on the long-term rates by Dybvig, Ingersoll and Ross (1996) .

When the initial frictionless model is arbitrage-free we proved that contingent claims prices remain the same in the limit of large quantities after the introduction of the "fixed costs." When the initial frictionless model contains arbitrage opportunities, we proved that the absence of arbitrage in the model with frictions is characterized by the existence of a nonnegative stateprice density (or equivalently by a martingale measure possibly with zero-weights) and that contingent claims prices are given (in the limit of large quantities) by this state-price density.

\section{ApPENDiX}

Proof of Proposition 2 The direct implication is immediate and using the fact that $\lim _{\lambda \rightarrow \infty} \frac{c^{\lambda \theta}\left(\sigma_{t}\right)}{\lambda} \rightarrow$ 0 it suffices to multiply the considered portfolio by a sufficiently large constant in order to establish the converse implication.

Proof of Proposition 3 As usual, this result is a direct application of Farkas-Minkowski Lemma.

\section{An example of utility maximization with zero state price densities in some states}

Let us consider a financial market where two securities denoted by A and B can be traded at two dates 0 and 1 and in two possible states of the world (with equal probability) $s_{1}$ and $s_{2}$ at date 1 (security A has a value of 1 at date 0 and a value of 1 or 2 at date 1 in state $s_{1}$ or $s_{2}$ respectively and security B is always worth one unit of account). We consider an agent, who, endowed with an initial wealth $w$ and a CARA utility function with an absolute risk aversion $\alpha=1$, tries to maximize his expected utility from terminal wealth. Furthermore, we assume that a transaction fee $c^{\theta}=\frac{1+2|\theta|}{2+2|\theta|}$ is paid at date 0 only if the agent trades a quantity $\theta \neq 0$ of asset $A$ at date 0 . Clearly, the transaction costs increase with the quantity traded and 
are bounded above and bounded away from zero for $\theta \neq 0$. It is easy to see that an optimal trade should satisfy the condition $\theta \geq 0$ and that it leads then to a terminal wealth equal to ${ }^{9}$ $\left(w-c^{\theta}, w+\theta-c^{\theta}\right)$. If $\theta$ is large enough, we have $w-c^{\theta} \approx w-1, w+\theta-c^{\theta} \approx w+\theta-1$ and any marginal increase of the traded quantity from $\theta$ to $\theta+\delta \theta$ would lead to a move of the utility level from $u$ to $u+\delta u$ with $\delta u=-\frac{1}{4} \exp \left(-w+c^{\theta}\right) \frac{1}{(1+\theta)^{2}}+\frac{1}{2} \exp \left(-w-\theta+c^{\theta}\right)\left(1-\frac{1}{2(1+\theta)^{2}}\right) \approx$ $-\frac{\exp (-w+1)}{4(1+\theta)^{2}}+\frac{1}{2} \exp (-w-\theta-1)<0$ for $\theta$ large enough. Furthermore, it is easy to see that for $\theta>-\ln \left(2 \exp \left(-c^{\theta}\right)-1\right)$, the utility level associated to that trade is higher than the utility level associated with no trade. In particular, for $\theta>-\ln \left(2 \exp \left(-\frac{1}{2}\right)-1\right) \approx 1.55$, it is better to trade. Hence, in such a framework our agent chooses to trade at a given optimal level $\theta^{*}$ such that $0<\theta^{*}<\infty$.

Proof of Proposition 5 If there exists an arbitrage portfolio $\theta$ as in Definition 1 between a node $\sigma_{t}$ and its successors, it suffices to consider the dynamic strategy that consists in buying the portfolio $\theta$ at the node $\sigma_{t}$, and investing the "profits" in bonds.

Conversely suppose that there is a dynamic arbitrage opportunity described by a portfolio $\theta$ then there exists a node $\sigma_{t}$ such that $\theta\left(\sigma_{t}\right) \cdot Z\left(\sigma_{t}\right)<0$. If $\theta\left(\sigma_{t}\right) \cdot Z\left(\sigma_{t+1}\right) \geq 0$ for all $\sigma_{t+1} \in f\left(\sigma_{t}\right)$, then according to Proposition 1 we have an arbitrage portfolio at $\sigma_{t}$; but if $\theta\left(\sigma_{t}\right) \cdot Z\left(\sigma_{t+1}\right)<0$ for some $\sigma_{t+1} \in f\left(\sigma_{t}\right)$, then using the self-financing condition, we have $\theta\left(\sigma_{t+1}\right) \cdot Z\left(\sigma_{t+1}\right)<0$; we start again from the node $\sigma_{t+1}$ and there must exist a date $\tau$ and a node $\sigma \in \Sigma_{\tau}$ such that $\theta(\sigma) \cdot Z(\sigma)<0$ and $\theta \cdot Z \geq 0$ on $f(\sigma)$ in order to obtain at the end $\theta \cdot Z \geq 0$.

Proof of Theorem 6 If there is no arbitrage opportunity it suffices to multiply the different state-price densities obtained at each node between that node and its successors starting from $\sigma$ to construct a nonnegative state-price density compatible with the asset prices.

\footnotetext{
${ }^{9}$ If $\theta=0$ then there is no trade and no transaction cost. We define then $c^{0}$ by $c^{0}=0$.
} 
Conversely, for a given date $i$-node $\sigma$, it suffices to consider the state-price density $\left(\pi_{\rho}\right)_{\rho \in f(\sigma)}$ defined on the successors of $\sigma$ by $\pi_{\rho}=\pi^{\sigma}(\rho)$. This state-price density characterizes the absence of arbitrage opportunities between $\sigma$ and its successors for every $\sigma$ and then the absence of dynamic arbitrage opportunities.

Proof of Proposition 8 It is easy to see that for all $m$ in $M$,

$$
\lim _{\lambda \rightarrow+\infty} \frac{p_{s}(\lambda m)}{\lambda}=\lim _{\lambda \rightarrow+\infty} \frac{-p_{s}(-\lambda m)}{\lambda}=l(m)
$$

Since there is no arbitrage, we must have $p(m) \geq-p(-m)$ so that

$$
-\pi_{s}(-m) \leq-p(-m) \leq p(m) \leq \pi_{s}(m),
$$

and the price functional $p$ can be written as the sum of a linear functional and of a "fixed cost" function, i.e. for all $m, p(m)=l(m)+c(m)$ where $\frac{c(\lambda m)}{\lambda} \rightarrow_{\lambda \rightarrow \infty} 0$. The case of bounded "fixed costs structures" can be treated similarly.

If we assume that $p(\lambda m) \leq \lambda[p(m)]$, it is easy to see that the "fixed cost" function is nonnegative. Furthermore, for all frictionless self-financing dynamic portfolio $\theta$,

$$
l\left(\left(\theta\left(\sigma_{T}\right) \cdot Z\left(\sigma_{T}\right)\right)_{\sigma_{T} \in \Sigma_{T}}\right)=\sum_{\sigma_{T} \in \Sigma_{T}} \pi^{\Omega}\left(\sigma_{T}\right) \theta\left(\sigma_{T}\right) \cdot Z\left(\sigma_{T}\right),
$$

where $\pi^{\Omega}$ is any nonnegative state-price density like in Theorem 4 . 
Consequently, the fair price $p(m)$ associated with any attainable contingent claim $m$ is given by

$$
p(m)=\sum_{\sigma_{T} \in \Sigma_{T}} \pi^{\Omega}\left(\sigma_{T}\right) m\left(\sigma_{T}\right)+\mathrm{a} \text { "fixed cost" }
$$

\section{An example of nondecreasing long-term rate with nonmonotone finite horizon rates}

Let us assume that the sequence of short-term rates $r_{n}$ between date $t=n$ and $n+1$ is $\infty$ uniform. ${ }^{10}$ It is clear that $z\left(n, n^{\prime}\right)$ (resp. $\left.z(n, n+N)\right)$ can both increase or decrease when $n^{\prime}$ (resp. $n$ ) increases. However $\ln \left(1+z_{L}(n)\right)$ is the limit of the average of the $\ln \left(1+r_{n}\right)$ and is equal to $\frac{1}{2} \ln 2$. We have then that $z_{L}(n)$ is constant, independent of $n$ and equal to $\sqrt{2}-1$.

Proof of Proposition 9 Proceeding like in the frictionless case, we obtain that the absence of arbitrage with "fixed costs" is implied by the existence of $\pi \in[0,1]$, independent from $n$, and such that $\pi h(n)+(1-\pi) h^{*}(n)=1$ and $\frac{h(n)}{h^{*}(n)}=\left[\frac{h(1)}{h^{*}(1)}\right]^{n}$.

Indeed, in that case we get that the discounted process $X^{T}=\left(\frac{\nu(t, T)}{\beta_{t}}\right)_{t}$ satisfies for all $\sigma_{t} \in \Sigma_{t}$

$$
X_{t}^{T}\left(\sigma_{t}\right)=\pi X_{t+1}^{T}\left(\sigma_{t}, u\right)+(1-\pi) X_{t+1}^{T}\left(\sigma_{t}, d\right)
$$

and the prices do not depend on the chosen path.

The choice of $\pi=1$ (which, by Theorem 6, is licit in a "fixed costs" framework) leads to $h \equiv 1$ and $h^{*}(n)=\left[h^{*}(1)\right]^{n}$. As far as long-term rates are concerned, we have

\footnotetext{
${ }^{10} \mathrm{~A}$ sequence $\left(x_{n}\right)$ with values in $[0,1]$ is said to be $k$-uniform if for all rectangle $D=\prod_{i=1}^{k}\left[a_{i}, b_{i}\right]$ with $0 \leq a_{i} \leq b_{i} \leq 1, i=1, \cdots, k$, we have

$$
\lim _{n \rightarrow \infty} \frac{1}{n} \sum_{i=0}^{n-1} 1_{D}\left(\left(x_{k i}, x_{k i+1}, \cdots, x_{k(i+1)-1}\right)\right)=\left(b_{1}-a_{1}\right) \cdots\left(b_{k}-a_{k}\right) .
$$
}

A sequence is said to be $\infty$-uniform if it is $k$-uniform for all $k \geq 1$. 


$$
\begin{aligned}
1+z_{L}\left(t,\left(\sigma_{t-1}, u\right)\right) & =\lim _{T / \infty}\left[\nu\left(t, T,\left(\sigma_{t-1}, u\right)\right)\right]^{-\frac{1}{T-t}} \\
& =\lim _{T / \infty}\left[\frac{\nu\left(t-1, T, \sigma_{t-1}\right)}{\nu\left(t-1, t, \sigma_{t-1}\right)} h(T-t)\right]^{-\frac{1}{T-t}} \\
& =1+z_{L}\left(t-1, \sigma_{t-1}\right)
\end{aligned}
$$

and

$$
\begin{aligned}
1+z_{L}\left(t,\left(\sigma_{t-1}, d\right)\right) & =\lim _{T \nearrow \infty}\left[\nu\left(t, T,\left(\sigma_{t-1}, d\right)\right)\right]^{-\frac{1}{T-t}} \\
& =\lim _{T \nearrow \infty}\left[\frac{\nu\left(t-1, T, \sigma_{t-1}\right)}{\nu\left(t-1, t, \sigma_{t-1}\right)} h^{*}(1)^{T-t}\right]^{-\frac{1}{T-t}} \\
& =\frac{1}{h^{*}(1)}\left[1+z_{L}\left(t-1, \sigma_{t-1}\right)\right] \\
& <1+z_{L}\left(t-1, \sigma_{t-1}\right)
\end{aligned}
$$

Proof of Proposition 10 It suffices to take $H(n, \rho)$ equal to $a^{n}$ (resp. 1 , resp. $\frac{1}{a^{n}}$ ) if $\rho=u$ (resp. $\rho=m$, resp. $\rho=d$ ) where $a<1$ is a given constant. There exists $\pi=(0,1,0) \in[0,1]^{3}$, such that $\pi_{1} a^{n}+\pi_{2}+\pi_{3}\left(1 / a^{n}\right)=1$, and, with the notations of the previous proof, the process $X^{T}=\left(\frac{\nu(t, T)}{\beta_{t}}\right)_{t}$ satisfies

$$
X_{t}^{T}\left(\sigma_{t}\right)=\pi_{1} X_{t+1}^{T}\left(\sigma_{t}, u\right)+\pi_{2} X_{t+1}^{T}\left(\sigma_{t}, m\right)+\pi_{3} X_{t+1}^{T}\left(\sigma_{t}, d\right) .
$$

By Theorem 6 this model is arbitrage-free in a "fixed costs" framework. As far as long-term rates are concerned and using the same approach as in the previous proof, we obtain

$$
\begin{aligned}
1+z_{L}\left(t,\left(\sigma_{t-1}, u\right)\right) & =\frac{1}{a}\left[1+z_{L}\left(t-1, \sigma_{t-1}\right)\right] \\
& >1+z_{L}\left(t-1, \sigma_{t-1}\right) \\
1+z_{L}\left(t,\left(\sigma_{t-1}, u\right)\right) & =1+z_{L}\left(t-1, \sigma_{t-1}\right) \\
1+z_{L}\left(t,\left(\sigma_{t-1}, d\right)\right) & =a\left[1+z_{L}\left(t-1, \sigma_{t-1}\right)\right] \\
& <1+z_{L}\left(t-1, \sigma_{t-1}\right)
\end{aligned}
$$

Proof of Proposition 11 Let us take $p_{u}=\frac{\sigma_{1}^{2}-\frac{\mu}{l}\left(\left(\frac{\mu}{l}-\frac{1}{2} \tilde{\sigma}_{1}^{2}\right) \frac{T}{N}-\tilde{\sigma}_{1} \sqrt{\frac{T}{N}}\right)}{2 \tilde{\sigma}_{1}\left[\tilde{\sigma}_{1}+\left(\frac{\mu}{l}-\frac{1}{2} \tilde{\sigma}_{1}^{2}\right) \sqrt{\frac{T}{N}}\right]}, p_{m}=\frac{\tilde{\sigma}_{1}^{2}-\sigma_{1}^{2}+\left(\frac{\mu}{l}{ }^{2}-\frac{1}{4} \tilde{\sigma}_{1}^{4}\right) \frac{T}{N}}{\tilde{\sigma}_{1}^{2}-\left(\frac{\mu}{l}-\frac{1}{2} \tilde{\sigma}_{1}^{2}\right)^{2} \frac{T}{N}}$, 
$p_{d}=\frac{\sigma_{1}^{2}-\frac{\mu}{l}\left(\left(\frac{\mu}{l}-\frac{1}{2} \tilde{\sigma}_{1}^{2}\right) \frac{T}{N}+\tilde{\sigma}_{1} \sqrt{\frac{T}{N}}\right)}{2 \tilde{\sigma}_{1}\left[\tilde{\sigma}_{1}-\left(\frac{\mu}{l}-\frac{1}{2} \tilde{\sigma}_{1}^{2}\right) \sqrt{\frac{T}{N}}\right]}, q_{u}=1 / 4, q_{m}=1 / 2, q_{d}=1 / 4$, for some positive constant $\sigma_{1}<\tilde{\sigma}_{1}$ and for $N$ sufficiently large. In that case, it is easy to check that $P=\left(p_{u}, p_{m}, p_{d}\right)$ defines a probability and following Nelson and Ramaswamy (1990) our model is a discrete approximation of the Brennan and Schwartz (1979) model.

Let the risk-neutral probability $[\pi(i, j)]_{\substack{i=u, m, d \\ j=u, m, d}}$ be such that $\pi(m, m)=1$ and let $\pi$ denote the induced probability measure on the terminal states of the world. We then construct bond prices as the discounted expected value under the probability $\pi$ of the bond's terminal payoff, and obtain

$$
\nu\left(n, n^{\prime} ;(\ell, r)\right)=\left[\left(1+u_{n}^{\ell, r}\right) \ldots\left(1+u_{n^{\prime}-1}^{\ell, r}\right)\right]^{-1}
$$

where the sequence $u_{s}^{\ell, r}$ is defined inductively by

$$
\left\{\begin{array}{l}
u_{s+1}^{\ell, r}=u_{s}^{\ell, r} \exp \left\{\frac{a\left(\ell-u_{s}^{\ell, r}\right)}{u_{s}^{l, r}} \frac{T}{N}\right\} \\
u_{n}^{\ell, r}=r
\end{array}\right.
$$

By construction and by Theorem 6, the financial market consisting of these bonds is arbitragefree in a "fixed costs" setting and admits $r$ as short-term rate. Furthermore, we can verify that the long-term rate induced by these bond prices is given by $\ell$. Indeed, we have

$$
\ln \left(1+z\left(n, n^{\prime} ;(\ell, r)\right)\right)=\frac{1}{n^{\prime}-n} \sum_{k=0}^{n^{\prime}-n-1} \ln \left(1+u_{n+k}^{\ell, r}\right)
$$

and by a classical Cesaro argument we get

$$
\ln \left(1+z_{L}(n ;(\ell, r))\right)=\lim _{k \rightarrow \infty} \ln \left(1+u_{k}^{\ell, r}\right) .
$$


when this last limit exists. It is then easy to check that the limit of $u_{k}^{\ell, r}$ is equal to $\ell$ and we obtain then $z_{L}(n ;(\ell, r))=\ell$.

Proof of Proposition 12 For $M=\infty,\left(Z_{t}\right)_{t \in\{0, \cdots, T\}}$ is equal to $\left(W_{t}\right)_{t \in\{0, \cdots, T\}}$ and there is a unique probability measure that we shall denote by $P^{*}$ that makes $\left(Z_{t}\right)_{t \in\{0, \cdots, T\}}$ a martingale and it is given by the positive transition probability $p^{*}=\frac{1}{2}-\frac{1}{4} \delta$. By Proposition 8 any fair pricing rule is given by the sum of a "fixed cost" function and of the expected value with respect to $P^{*}$ (which corresponds to the option price in a frictionless setting).

It is easy to check that this model is also arbitrage free in a fixed-costs setting for $M<\infty$.

We turn then directly to pricing issues. There is a unique probability measure $P_{M}^{*}$ with nonnnegative weights which makes $\left(Z_{t}\right)$ a martingale. This probability is equal to the above mentioned probability measure (with positive weights) $P^{*}$ on the paths for which the bound $M$ is not reached and puts a zero weight on the paths for which the bound is reached and left. The process $\left(Z_{t}\right)$ has under $P_{M}^{*}$ the same distribution as the primitive price process absorbed at the upper bound $M$ (and denoted by $\tilde{W}_{T}$ ) under $P^{*}$. Thus any admissible price for a European call $x=\left(Z_{T}-K\right)_{+}$can be written as the sum of a fixed cost and of $E^{P_{M}^{*}}\left[\left(Z_{T}-K\right)_{+}\right]$which is equal to $E^{P^{*}}\left[\left(\tilde{W}_{T}-K\right)_{+}\right]$and corresponds to the price of a barrier option, where the barrier is set at $M$, in the classical binomial model where the risk-neutral probability is given by $P^{*}$.

Proof of Proposition 13 As in CIR or in Longstaff (1992) we look for a state-price density $\pi$ compatible with the no-arbitrage condition in a "fixed costs" setting such that the risk premium is equal to $\lambda$ for $r \neq 0$.

More precisely, we look for $\left.\pi=\left(\pi_{1}, \pi_{2}, \pi_{3}\right) \in\right] 0,1\left[^{3}\right.$, such that under $\pi$, the drift is given by the initial drift minus $\lambda r$ (as in CIR), and the variance remains unchanged (and given by 
$\left.\sigma^{2} r \frac{T}{N}\right)$. If $|\lambda|<\frac{a}{\sqrt{2}}, \sigma^{2} \geq \lambda^{2} \bar{r} \frac{T}{N}$, where $\bar{r}=\sup \left\{r_{0},\left(\frac{\sqrt{2} \sigma+\sqrt{2 \sigma^{2}+4 a^{2} l h^{2}}}{2 a h}\right)^{2}\right\}$, the solution is given by $\pi_{1}=\frac{1}{2}\left[\frac{\sigma^{2}+\frac{\lambda^{2} T r}{N}}{2 \sigma^{2}}-\frac{\lambda \sqrt{r}}{\sigma \sqrt{2}} \sqrt{\frac{T}{N}}\right], \pi_{3}=\frac{1}{2}\left[\frac{\sigma^{2}+\frac{\lambda^{2} T r}{N}}{2 \sigma^{2}}+\frac{\lambda \sqrt{r}}{\sigma \sqrt{2}} \sqrt{\frac{T}{N}}\right]$ and $\pi_{2}=1-\pi_{1}-\pi_{3}$. Furthermore, we want our model to have an absorbing barrier under the risk-neutral probability in order to generate the same prices as in Longstaff (1992). It suffices then to take, for $r=0$, $\left(\pi_{1}, \pi_{2}, \pi_{3}\right)=(0,0,1)$

Letting $\nu\left(r ; n, n^{\prime}\right)$ be given by

$$
\nu\left(r ; n, n^{\prime}\right)=E^{\pi}\left[\frac{1}{\prod_{s=n}^{n^{\prime}-1}\left(1+r_{s}\right)} \mid r\right]
$$

we obtain an arbitrage-free (with "fixed costs") term structure which satisfies $\nu\left(0 ; n, n^{\prime}\right)=1$ (as in Longstaff (1992)) and the market price of interest-rate risk is $\lambda$ (as in CIR and in Longstaff $(1992))$.

\section{References}

[1] Black, F. and M. Scholes. "The Pricing of Options and Corporate Liabilities." Journal of Political Economy, 81 (1973), 637-659.

[2] Brennan, M.J. "The Optimal Number of Securities in a Risky Asset Portfolio when there are Fixed Costs of Transacting." Journal of Financial and Quantitative Analysis, 10 (1975), 483-496.

[3] Brennan, M.J. and E.S. Schwartz. "A Continuous-Time Approach to the Pricing of Bonds." Journal of Banking and Finance. 3 (1979), 135-155. 
[4] Brown, S.J. and P.H. Dybvig. "The Empirical Implications of the Cox, Ingersoll, Ross Theory of the Term Structure of Interest Rates." Journal of Finance, 41 (1986), 617-632.

[5] Brown, R.H. and S.M. Schaefer. "The Term Structure of Real Interest Rates and the Cox, Ingersoll, Ross Model." Journal of Financial Economics, 35 (1994), 3-42.

[6] Cecchetti, S.G. "The Case of the Negative Nominal Interest Rates: New Estimates of the Term Structure of Interest Rates during the Great Depression." Journal of Political Economy, 96 (1988), 1111-1141.

[7] Chan, K.C., Karolyi, G.A., Longstall, F.A. and A.B. Sanders. "An Empirical Comparison of Alternative Models of the Short-Term Interest Rate." The Journal of Finance, 47 (1992), $1209-1227$.

[8] Cox, J.C., Ingersoll, J.E. and S.A. Ross. "A Theory of the Term Structure of Interest Rates." Econometrica, 53 (1985), 385-407.

[9] Cox, J., Ross, S. and M. Rubinstein. "Option Pricing: A Simplified Approach." J. Fin. Eco., 7 (1979), 229-261.

[10] Duffie, D. and R. Kan. "A Yield-Factor Model of Interest Rates." Mathematical Finance, 6 (1996), 379-406.

[11] Duffie, D. and T. Sun. "Transaction Costs and Portfolio Choice in a Discrete-Continuous Time Setting." J. Econ. Dyn. and Control, 14 (1990), 35-51.

[12] Dybvig, P., Ingersoll, J. and S. Ross. "Long Forward and Zero-Coupon Rates can Never Fall." J. Business, 69 (1996), 1-25. 
[13] Goldsmith, D. "Transaction Costs and the Theory of Portfolio Selection." Journal of Finance, 31 (1976), 1127-1139.

[14] Grossman, S. and G. Laroque. "Asset Pricing and Optimal Portfolio Choice in the Presence of Illiquid Durable Consumption Goods." Econometrica, 58 (1990), 25-51.

[15] Ho, T.S.Y. and S.-B. Lee. "Term Structure Movements and Pricing Interest Rate Contingent Claims." Journal of Finance, 41 (1986), 1011-1029.

[16] Jouini, E., and H. Kallal. "Martingales and Arbitrage in Securities Markets with Transaction Costs." Journal of Economic Theory, 66 (1995), 178-197.

[17] Jouini E., Kallal H. and C. Napp. "Arbitrage in Financial Markets with Fixed Costs." Journal of Mathematical Economics, 35 (2001), 197-221.

[18] Leape, J. "Taxes and Transaction Costs in Asset Market Equilibrium." Journal of Public Economics, 33 (1987), 1-20.

[19] Levy, H. "Equilibrium in an Imperfect Market : a Constraint on the Number of Securities in a Portfolio." American Economic Review, 68 (1978), 643-658.

[20] Longstaff, F.A. "Multiple Equilibria and Term Structure Models." Journal of Financial Economics, 32 (1992), 333-344.

[21] Luttmer, E.G.J. "What Level of Fixed Costs can Reconcile Consumption and Stock Returns?" The Journal of Political Economy, 107(5) (1999), 969-997.

[22] Mayshar, J. "Transaction Costs in a Model of Capital Market Equilibrium." Journal of Political Economy, 87 (1979), 673-700. 
[23] Mayshar, J. "Transaction Costs and the Pricing of Assets." Journal of Finance, 36 (1981), 583-597.

[24] Morton, A. and S. Pliska. "Optimal Portfolio Management with Fixed Transaction Costs." Math. Fin., 5 (1995), 337-56.

[25] Nelson, D. and K. Ramaswamy. "Simple Binomial Processes as Diffusion Approximations in Financial Models." Review of Financial Studies, 3 (1990), 393-430.

[26] Sondermann, D. "Currency Options: Hedging and Social Value." Eur. Econ. Rev., 31 (1987), 246-256.

[27] Sondermann, D. "Option Pricing with Bounds on the Underlying Securities." Bankpolitik, Finanzielle Unternehmensführung and die Theorie der Finanzmärkte. Berlin, Duncker\&Humblot (1988).

[28] Viard, A.D. "The Asset Pricing Effects of Fixed Holding Costs : an Upper Bound." Journal of Financial and Quantitative Analysis, 30(1) (1995), 43-59. 